\title{
Digital Signal Processing for Hearing Instruments
}

\section{Heinz G. Göckler (EURASIP Member), ${ }^{1}$ Henning Puder, ${ }^{2}$ Hugo Fastl, ${ }^{3}$ Sven Erik Nordholm, ${ }^{4}$ Torsten Dau, ${ }^{5}$ and Walter Kellermann ${ }^{6}$}

\author{
${ }^{1}$ Digital Signal Processing Group, Ruhr-Universität Bochum, 44780 Bochum, Germany \\ ${ }^{2}$ Siemens Audiologische Technik GmbH, 91058 Erlangen, Germany \\ ${ }^{3}$ Institute for Human-Machine Communication, Technische Universität München, 80333 München, Germany \\ ${ }^{4}$ Signal Processing Laboratory, Western Australian Telecommunications Research Institute (WATRI), Crawley, \\ WA 6009, Australia \\ ${ }^{5}$ Centre for Applied Hearing Research, Acoustic Technology, Department of Electrical Engineering, \\ Technical University of Denmark, 2800 Kongens Lyngby, Denmark \\ ${ }^{6}$ Multimedia Communications and Signal Processing, Universität Erlangen-Nürnberg, 91058 Erlangen, Germany
}

Correspondence should be addressed to Heinz G. Göckler, goeckler@nt.rub.de

Received 26 October 2009; Accepted 26 October 2009

Copyright (c) 2009 Heinz G. Göckler et al. This is an open access article distributed under the Creative Commons Attribution License, which permits unrestricted use, distribution, and reproduction in any medium, provided the original work is properly cited.

Hearing, as a prerequisite of listening, presumably represents the most important pillar of human's ability to communicate with each other. Hence, engineers of all denominations, physicists, and physicians have always been creative both to improve the environmental conditions of hearing and to ameliorate the individual hearing capability.

Digital signal processing for hearing instruments has been an active field of research and industrial development for more than 25 years. As a result, these efforts have eventually paid off and, thus, opened big markets for digital hearing aids and cochlear implants which, in turn, promote and accelerate related research and development. Certainly, the present state-of-the-art of hearing instruments has highly profited from efficient small size technology with very low power consumption mainly developed for portable communication equipment, advanced digital filtering and filter banks, as well as speech processing and enhancement devised for modern speech transmission and recognition. Moreover, these examples of cross-fertilisation exploiting synergies are continuing and expanding on a large scale.

To promote the aforementioned cross-fertilisation, the goal of this special issue on Digital Signal Processing for Hearing Instruments (DSPHI) has been to collect and present the latest state-of-the-art research in signal processing methods and algorithms used in or suitable for hearing instruments, as well as in related design approaches and implementations. Just four years ago, in this journal a first special issue on this topic was published, underlining the importance and activity of this challenging field of research: EURASIP Journal of Applied Signal Processing 2005:18.

This special issue on DSP for Hearing Instruments gathers 16 articles. Again, it reflects various aspects of multiple disciplines needed for the treatment of hearing impairment. In fact, the included papers address a variety of approaches, algorithms, and designs applicable to hearing instruments that are all amenable to DSP implementation.

The papers in this issue are organized according to their focus of research, since some of these contributions encompass multiple topics. To this end, we have combined the contributions to the following classes: (i) head-related transfer functions and filter banks (2 articles) embracing all DSP inherent to hearing instruments, (ii) noise reduction and speech enhancement (8 articles) encompassing combinations with other algorithms, beamforming, and related measurement procedures, (iii) feedback cancellation (1 article), (iv) sound classification (2 articles), (v) cochlear implants (2 articles), and (vi) personal hearing system (1 article).

The paper entitled "Database of multichannel in-ear and behind-the-ear head-related and binaural room impulse responses" (H. Kayser et al.) offers a database of head-related impulse responses (HRIRs) and binaural room impulse responses (BRIRs). These data are measured with threechannel behind-the-ear (BTE) hearing aids and an inear microphone at both ears of a human head-and-torsosimulator. The database provides a tool for the evaluation of multichannel hearing aid algorithms in hearing aid research. 
The presented database allows for a realistic construction of simulated sound fields for the investigation of hearing instruments.

Another paper entitled "A novel approach to the design of oversampling low-delay complex-modulated filter bank pairs" (T. Kurbiel et al.) proposes a noniterative twostep procedure for the design of the prototype filters of oversampling uniform complex-modulated FIR filter bank pairs that applies two convex objective functions: (i) design of the analysis prototype filter for minimum group delay, and (ii) design of the synthesis prototype filter such that the filter bank pair approximates a linear-phase allpass function. Both aliasing and imaging distortion is controlled by refined frequency-dependent stopband constraints tailored to the actual oversampling factor. The derivation of the constraints, however, will be published elsewhere.

In the paper entitled "Incorporating the conditional speech presence probability in multi-channel Wiener filter based noise reduction in hearing aids" (K. Ngo et al.) the conditional speech presence probability (SPP) is applied to the speech distortion weighted multi-channel Wiener filter (SDW-MWF) which focuses on the improvement of the noise reduction performance of the traditional SDW-MWF. This approach exploits sparseness of speech signals, that is, the fact that speech may not be present at all times and at all frequencies, which is not taken into account by a typical SDW-MWF. This leads to less noise reduction in speech dominant segments leading to less speech distortion and a higher noise reduction during speech absence. The noise reduction performance of the proposed method is evaluated and compared with a typical SDW-MWF.

In the paper entitled "Improved reproduction of stops in noise reduction systems with adaptive windows and nonstationarity detection" (D. Mauler et al.) a novel block-based noise reduction system is proposed which focuses on the preservation of transient sounds like stops or speech onsets. The key idea of the proposed system is the detection of nonstationary input data. Depending on that decision a pair of analysis-synthesis windows is adaptively selected which either provides high temporal or high spectral resolution. Furthermore, the decision-directed approach to estimate the a priori SNR is modified such that speech onsets are tracked more instantaneously without sacrificing performance in stationary signal regions.

In the paper entitled "Robust distributed noise reduction in hearing aids with external acoustic sensor nodes" (A. Bertrand et al.) the benefit of using external acoustic sensor nodes for noise reduction in hearing aids is demonstrated in a simulated acoustic scenario with multiple sound sources. A distributed adaptive node-specific signal estimation (DANSE) algorithm with reduced bandwidth and diminished computational load is evaluated and compared with the noise reduction performance of centralized multichannel Wiener filtering (MWF).

The paper entitled "Synthetic stimuli for the steady-state verification of modulation-based noise reduction systems" (J. G. Lamm et al.) provides partial verification of the performance of hearing instruments by measuring the properties of their noise reduction systems. Synthetic stimuli with their potential of adaptability to the parameter space of the noise reduction system are proposed as test signals. The article presents stimuli for steady-state measurements of modulation-based noise reduction systems.

The paper entitled "The acoustic and perceptual effects of series and parallel processing" (M. C. Anderson et al.) explores how spectral subtraction and dynamic-range compression gain modifications affect temporal envelope fluctuations in parallel and serial system configurations. In parallel processing, both algorithms derive their gains from the same input signal. In serial processing, the output from the first algorithm forms the input to the second algorithm. Acoustic measurements show that the parallel arrangement produces higher gain fluctuations and, thus, gives rise to higher variations of the temporal envelope than with the serial configuration. Intelligibility tests both for normalhearing and for hearing-impaired listeners demonstrate that (i) parallel processing leads to significantly poorer speech understanding than that with an unprocessed (UNP) signal and with the serial arrangement and (ii) serial processing and UNP yield similar results.

The paper entitled "Low delay noise reduction and dereverberation for hearing aids" (H. W. Löllmann et al.) presents a new, low signal delay algorithm for single-channel speech enhancement, which concurrently achieves suppression of late reverberant speech and background noise. It is based on a generalized spectral subtraction rule which depends on the variance both of the late reverberant speech and of the background noise. Compared to the commonly used postfiltering, which solely performs noise reduction, both the subjective and the objective speech quality is significantly improved by the proposed algorithm.

The paper entitled "A computational auditory scene analysis-enhanced beamforming approach for sound source separation" (L. A. Drake et al.) describes a novel approach to sound source separation, which achieves better signal separation performance by combining complementary techniques. The method concurrently exploits features of the sound sources and, by applying multimicrophone beamforming, the local separation of the sources, where both attributes are independent of each other.

The paper entitled "Rate-constrained beamforming in binaural hearing aids" (S. Srinivasan et al.) addresses hearing aid systems, where the left and right ear devices collaborate with each other. Binaural beamforming for hearing aids requires an exchange of microphone signals between the two devices over a wireless link. In this contribution, two issues are investigated: which multiple microphone signals to transmit from one ear to the other, and at what bit-rate. Obviously, the second problem is relevant as the capacity of the wireless link is limited by stringent power consumption constraints.

The paper entitled "Combination of adaptive feedback cancellation and binaural adaptive filtering in hearing aids" (A. Lombard et al.) studies a system combining adaptive feedback cancellation and adaptive filtering connecting inputs from both ears for signal enhancement in hearing aids. Such a binaural system is analysed in terms of system stability, convergence of the algorithms, and possible 
interaction effects. As major outcomes of this study, a new stability condition adapted to the considered binaural scenario is presented, some commonly used feedback cancellation performance measures for the unilateral case are adapted to the binaural case, and possible interaction effects between the algorithms are identified.

The paper entitled "Influence of acoustic feedback on the learning strategies of neural network-based sound classifiers in digital hearing aids" (L. Cuadra et al.) investigates the feasibility of using different feedback-affected sound databases, and a variety of learning strategies of neural networks to reduce the probability of erroneous classification. The experimental work basically shows that the proposed methods support the neural network-based sound classifiers in reducing its error probability by more than $18 \%$.

The paper entitled "Analysis of the effects of finite precision in neural network-based sound classifiers for digital hearing aids" (R. Gil-Pita et al.) deals with the implementation of sound classifiers with restricted resources such as finite arithmetic and a limited number of instructions per second. This contribution focuses on exploring (i) the most appropriate quantisation scheme for digitally implemented neural network-based classifiers, and (ii) the most adequate approximation for its activation function. Experimental work shows that the optimized finite precision neural network-based classifier achieves the same performance as the corresponding full precision approach and, hence, considerably reduces the computational load.

The paper entitled "Signal processing strategies for cochlear implants using current steering" (W. Nogueira et al.) presents new concepts of sound processing strategies in cochlear implants. In contemporary systems, the audio signal is decomposed into different frequency bands, each assigned to one electrode. Thus, pitch perception is limited by the number of physical electrodes implanted into the cochlea and by the wide bandwidth assigned to each electrode. Two new sound processing strategies are presented here, where current is simultaneously delivered to pairs of adjacent electrodes. By steering the locus of stimulation to sites between the electrodes, additional pitch percepts can be generated. Results from measures of speech intelligibility, pitch perception, and subjective appreciation of sound obtained with the two current steering strategies and a standard strategy are compared in 9 adult cochlear implant users. The benefit is shown to vary considerably while the mean results show similar performance with all three strategies.

The paper entitled "Prediction of speech recognition in cochlear implant users by adapting auditory models to psychophysical data" (S. Stadler et al.) addresses the large variability of speech recognition by users of cochlear implants under noisy conditions. From the many factors involved, discrimination of spectral shapes is studied in detail and incorporated in a model. Speech recognition performance is significantly correlated to model data for spectral discrimination. Hence, it is suggested to use the framework presented for simulating effects of changing the encoding strategy implemented in cochlear implants.
Finally, the paper entitled "The Personal Hearing System-a software hearing aid for a personal communication System" (G. Grimm et al.) describes an architecture of a personal communication system (PCS) that integrates audio communication and hearing support for elderly and hearing-impaired persons. The concept applies a central processing unit connected to audio headsets via a wireless body area network (WBAN). To demonstrate the feasibility of the concept, a prototype PCS is implemented on a small notebook computer with a dedicated audio interface in combination with a mobile phone. The prototype PCS integrates a hearing aid applying binaural coherence-based speech enhancement, telephony, public announcement systems, and home entertainment.

\author{
Heinz G. Göckler \\ Henning Puder \\ Hugo Fastl \\ Sven Erik Nordholm \\ Torsten Dau \\ Walter Kellermann
}

